\title{
Erratum
}

\section{Erratum to: A Trotter product formula for gradient flows in metric spaces}

PhilipPe ClÉment AND JAN MAAS

\section{Erratum to: J Evol Equ 11 (2011), 405-427 DOI 10.1007/s00028-010-0096-5}

In the original publication, the statement of Theorem 1.1 contains a typo which is corrected here. The factor $\frac{1}{2}$ should be removed from the left-hand side of equations (1.4), (1.5), and in the displayed formula between these equations. For example, (1.4) should be replaced by

$$
\frac{d}{d t} d^{2}(u(t), y) \leq \varphi(y)-\varphi(u(t))
$$

The rescaled function $v:\left[0, T_{*} / 2\right] \rightarrow X$ defined by $v(t)=u(2 t)$ satisfies the equations which have been stated for $u$.

If the factor $\frac{1}{2}$ is omitted, the theorem is correct. The proof is correct as stated and shows convergence to the correct equation, as can be seen, for example, in the last displayed formula of the proof on page 418 .

Further errors that need to be amended are given below:

- On line 8 of page $413,\left[t_{j-1}, t_{j}\right)$ should be replaced by $\left[t_{\mathbf{h}}^{j-1}, t_{\mathbf{h}}^{j}\right.$ ).

- The second line of the proof to Lemma 3.1 should be: "For the convenience of the reader, we provide a simple direct proof of (1) in the case that $\mu \ll \mathscr{L}^{d}$."

The second named author is supported by Rubicon subsidy 680-50-0901 of the Netherlands Organisation for Scientific Research (NWO).

The online version of the original article can be found under doi:10.1007/s00028-010-0096-5. 
Ph. Clément

Delft Institute of Applied Mathematics, Delft University of Technology, P.O. Box 5031, 2600 GA, Delft,

The Netherlands

E-mail:ppjeclement@aim.com

\section{J. Maas}

Institute for Applied Mathematics, University of Bonn,

Endenicher Allee 60, 53115 Bonn, Germany

E-mail:maas@iam.uni-bonn.de 